\title{
Validez de contenido, de constructo y confiabilidad del Dental Anxiety Scale en adultos chilenos.
}

\section{Content validity, construct validity and reliability of the Dental Anxiety Scale in Chilean adults.}

\author{
Matías Ríos-Erazo ${ }^{1}$, Braulio Santibañez ${ }^{2}$, Paula van Treek \\ Andrea Herrera-Ronda ${ }^{1 *}$, Gonzalo Rojas-Alcayaga ${ }^{1}$
}

\author{
1. Área de Ciencias del Comportamiento, Instituto \\ de Investigación en Ciencias Odontológicas, \\ Facultad de Odontología, Universidad de Chile. \\ Chile \\ 2. Estudiante de Pregrado Carrera de Odontología, \\ Facultad de Odontología, Universidad de Chile. \\ Chile \\ * Correspondencia Autor: Andrea Herrera | \\ Dirección: Olivos 943, Independencia, Santiago, \\ Chile | Teléfono: +562 29781844 | E-mail: \\ aherrera@odontologia.uchile.cl \\ Trabajo recibido el 02/10/2019. \\ Aprobado para su publicación el 24/11/2019
}

\begin{abstract}
RESUMEN
Introducción: La ansiedad dental impacta profundamente en la salud oral de un paciente, determinando el pronóstico y adherencia a tratamiento, sin embargo, existe poca información de instrumentos validados que evalúen este constructo. El objetivo de este estudio es evaluar las propiedades psicométricas del Dental Anxiety Scale versión en español en adultos en servicios de atención primaria de la Región Metropolitana, Chile. Material y método: Estudio transversal descriptivo aplicado a 169 adultos acompañantes de un servicio de atención primaria. La validez de contenido se determinó con metodología Delphi, la validez de constructo a través del análisis factorial confirmatorio y la confiabilidad a través de análisis de consistencia interna mediante Alfa de Cronbach. Resultados: El juicio de expertos no modificó la versión en español. El análisis factorial confirmó la presencia de un solo factor, el cual explicó el $65,44 \%$ de la varianza, mientras que la confiabilidad arrojó un alfa de Cronbach de 0,822, considerado bueno. Discusión: EI Dental Anxiety Scale presentó una adecuada validez de contenido, validez de constructo y buena confiabilidad. Se recomienda su uso para la medición de ansiedad dental en adultos que acuden a servicios de atención primaria en la Región Metropolitana.
\end{abstract}

PALABRAS CLAVE

Ansiedad dental; Validez; Confiabilidad; Salud oral.

Int. J. Inter. Dent Vol. 13(1); 09-12, 2020.

\begin{abstract}
Introduction: Dental anxiety can strongly affect oral health, modifying the prognosis and treatment's adherence. The level of dental anxiety has to be considered when proposing a treatment plan. The aim of this study is to measure the psychometric properties of the Dental Anxiety Scale in the Spanish version, applied to adults in primary health institutions of the Metropolitan Region, Chile Methods: 169 adults were recruited. To determine content validity, the Delphi method was used; the confirmatory factor analysis was used to demonstrate the construct validity. Reliability was measured in terms of internal consistency with the Cronbach's alpha coefficient. Results: The experts did not modify the Spanish version of DAS. The factorial analysis confirmed that there was a single factor that accounted for $65,44 \%$ of variance. A 0,882 Cronbach's alfa is considered as good reliability. Conclusion: Dental Anxiety Scale presents an appropriate construct and content validity, and a good reliability. It is a proper instrument to be used in adults in primary health care centers in the Metropolitan Region.
\end{abstract}

\section{KEY WORDS}

Dental anxiety, Validation, Reliability, Oral health.

Int. J. Inter. Dent Vol. 13(1); 09-12, 2020.

\section{INTRODUCCIÓN}

En Odontología actualmente se trabaja implementando un modelo de atención integral con enfoque familiar y comunitario(1) $^{(1)}$ y dentro de esta visión integral es de relevancia identificar las actitudes y conductas que influyen en la salud dental de las personas, variables imprescindibles si se quiere dar una óptima e íntegra atención al paciente ${ }^{(2)}$. Muchas personas poseen impedimentos individuales para acceder a la atención odontológica integral, los cuales en ocasiones están relacionados a una mala experiencia durante su primera atención o experiencias de atenciones desagradables y/o dolorosas de otras personas que son integradas como propias, generando un estado de temor y ansiedad frente a la atención dental ${ }^{(3)}$.

La ansiedad dental (AD) se define como una experiencia desagradable que varía entre las personas, de carácter subjetivo, que involucra un componente psicológico, fisiológico y motor, y que se tiene como respuesta antes, durante o después de la atención dental(4).

Ésta es una problemática que ocurre a nivel mundial, en donde los pacientes evitan acudir a un centro de atención dental hasta la última instancia. Diversos estudios informan sobre la prevalencia de la $A D$ en 
el mundo(5). En Alemania la prevalencia fue del $40 \%$ de la población ${ }^{(6)}$ mientras que en Noruega es de al menos un $16 \%{ }^{(5)}$. En Australia, la prevalencia llegó a un $31,2 \%{ }^{(7)}$ con representatividad nacional. Mientras tanto en Brasil, un $24 \%$ de la población también la presentaba(8). En Chile, las cifras van entre el $23,7 \%$ de los pacientes que acudieron a un servico de urgencia ${ }^{(9)}$ hasta el $37,9 \%$ de los pacientes en atención primaria de un centro de la Región Metropolitana ${ }^{(10)}$.

La ansiedad dental tiene una influencia directa en la salud oral(6). Pacientes con este problema presentan malos hábitos de higiene oral como la falta de cepillado, mala alimentación, tabaquismo, así como baja motivación para cuidar su condición oral ${ }^{(11)}$. Esto lleva a que el pronóstico de los procedimientos realizados y la adherencia al tratamiento por parte de los pacientes sea desalentador ${ }^{(12,13)}$. La reducción de la ansiedad dental en pacientes adultos aumentaría la asistencia de estos al dentista de manera más regular ${ }^{(11)}$.

Para cuantificar el grado de $A D$ en un paciente, se utilizan con frecuencia cuestionarios como el Dental Anxiety Scale (DAS) ${ }^{(14)}$, Modified Dental Anxiety Scale (MDAS) ${ }^{(15)}$ y el Dental Fear Scale ${ }^{(16)}$. El MDAS es una versión modificada del DAS, que incluye una pregunta referente al uso de anestesia local y así incluye el uso de agujas en odontología(15). Sin embargo, se consideró que este ítem interfiere en la real clasificación de $A D$, pues los procedimientos que requieren uso de agujas no son exclusivos a la atención dental, lo que podría llevar a sobreestimar el grado de ansiedad dental. Es también relevante considerar, que no todos los procedimientos dentales requieren del uso de anestesia local, sobretodo el tratamiento preventivo, foco principal de los servicios de atención primaria en salud.

EI DAS posee varias ventajas en la aplicación, como son su facilidad de respuesta, el tener un formato corto y la interpretación de los resultados. EI DAS es uno de los instrumentos más utilizados en el mundo para el estudio de la ansiedad dental(4,17) y fue el seleccionado para identificar la $A D$ en este estudio.

Si bien en Chile se utilizan estos cuestionarios, no existen datos de validación en el país, por lo que resulta importante contar con un instrumento confiable y con propiedades psicométricas adecuadas a la población local.

El proceso de validación de un cuestionario consta de distintas etapas. En primera instancia, el cuestionario debe estar validado en su idioma original y luego debe ser traducido al idioma que se desea validar, adaptándolo culturalmente pero manteniendo sus propiedades, por lo que se evalúan las características psicométricas de validez y confiabilidad ${ }^{(18)}$.

Considerando lo anteriormente mencionado, es que la presente investigación tiene como objetivo determinar la validez de contenido, validez de constructo y confiabilidad de la escala de ansiedad dental (DAS) en población consultante de centros de atención primaria de la Región Metropolitana.

\section{MATERIAL Y MÉTODO}

Se define un diseño de estudio no experimental, transversal descriptivo. La muestra, de tipo no probabilística, la componen hombres y mujeres, mayores de 18 años, acompañantes de pacientes del servicio dental de los CESFAM Recreo y Bellavista, de las comunas de San Miguel y La Florida respectivamente. Los acompañantes firmaron un consentimiento informado de participación en el estudio y luego se les aplicó la escala de ansiedad dental (DAS) en su versión traducida al español (16).

Entre los criterios de inclusión se consideraron a hombres y mujeres de nacionalidad chilena, mayores de 18 años y que sean acompañantes de pacientes de 6 años de edad beneficiarios del programa de salud oral integral en servicios de atención primaria de la Región Metropolitana. Se excluyó a adultos con déficits cognitivos, trastornos neurológicos orgánicos o trastornos de ansiedad generalizada (relatado al momento de aplicación del cuestionario). Esta investigación fue evaluada y aprobada por el comité ético y científico de la Facultad de Odontología de la Universidad de Chile. El presente estudio se enmarca dentro del proyecto FONIS SA11/2025, llamado "Impacto en la adherencia a tratamiento de una intervención cognitivo-conductual para disminuir ansiedad dental en niños y niñas de 6 años beneficiarios del programa de salud oral integral en servicios de atención primaria de la Región Metropolitana" realizado durante el año 2011

Esta escala de medición de la ansiedad dental cuenta con una traducción al español ${ }^{(16)}$ y ha sido el instrumento más usado internacionalmente en los estudios empíricos para evaluar la ansiedad dental(17), lo que permitiría realizar estudios comparativos.

Para obtener las propiedades psicométricas del DAS se deben cumplir con distintas fases: en primera instancia el documento debe estar validado en su idioma original(14) y tener una adaptación al idioma español(16). Luego, se debe comprobar que el instrumento mantiene sus propiedades para ser aplicado en población adulta chilena, evaluando las características psicométricas de validez y confiabilidad ${ }^{(18)}$, siendo estas últimas dos fases, los objetivos de la presente investigación.

EI DAS busca determinar el nivel de ansiedad dental que el entrevistado presenta en distintos contextos clínicos y no clínicos. Las preguntas tienen 5 alternativas de respuestas que varían de 1 (no ansioso) a 5 (extremadamente ansioso), con puntajes totales que van de 4 a 20 puntos $^{(14)}$. La encuesta utilizada puede verse más detalladamente en Anexo 1.

Para efectos de esta investigación, la validez de contenido se obtuvo a través de la metodología Delphi, que busca determinar el grado de consenso o acuerdo de los especialistas sobre el problema planteado ${ }^{(19)}$. Así, el cuestionario de ansiedad dental DAS se sometió a juicio de 4 expertos, 2 de ellos con título de magister en psicología, 1 poseía la especialidad en trastornos témporomandibulares y dolor orofacial y el último doctorado en psicología, además de tener el título de cirujanodentista; todos seleccionados por su amplio conocimiento y experiencia en el tema. Los expertos fueron contactados vía correo electrónico, y evaluaron la traducción en idioma español en cuanto al lenguaje utilizado, la redacción, su congruencia y pertinencia. Las respuestas, correcciones y anotaciones de los expertos se recogieron vía correo electrónico. Por último, los expertos se reunieron para comentar sus juicios y respuestas, llegando así a un consenso.

Por otra parte, la validez de constructo de la escala de ansiedad dental DAS se determinó mediante un análisis factorial confirmatorio (AFC) utilizando el método de correlación de variables, realizando el test de Bartlett y el índice de Kaiser Meyer Olkin. Por último, la medición de la confiabilidad se realizó a través del análisis de consistencia interna mediante Alfa de Cronbach $^{(20)}(\alpha)$, utilizando en todos éstos el software SPSS versión 21.0 para los análisis estadísticos. Se trabajó con un error de significancia del $5 \%$.

\section{RESULTADOS}

La muestra obtenida estuvo compuesta por un total de 169 pacientes adultos, de los cuales 150 eran mujeres y 19 eran hombres, siendo la edad promedio 37,3 años $(D . E .=11,00)$. La distribución según CESFAM fue de $43,8 \%$ en Recreo y de $56,2 \%$ en Bellavista. La Tabla 1 representa las variables sociodemográficas de los adultos encuestados.

En relación a la pregunta 1 del cuestionario de ansiedad dental DAS ¿Cómo se siente de saber que tiene una cita con el dentista?, la mayor parte $(37,7 \%)$ de los encuestados refirió que le agrada asistir pues lo considera una experiencia gratificante, seguido de un $21,3 \%$ que dijo estar temeroso pues lo considera una experiencia desagradable y dolorosa.

En relación a la pregunta 2, ¿Cómo se siente cuando está esperando su turno en la sala de espera?, un 39,6\% (67 personas) manifestaron estar relajadas y un 9,5\% (16 personas) refirió estar muy ansioso.

En relación a la pregunta ¿Cómo se siente cuando está en el sillón esperando que el dentista saque los instrumentos con que examinará sus dientes?, un $26,6 \%$ (45 personas) refirió estar tenso y un $23,1 \%$ (39 personas) dijeron sentirse relajadas.

En relación a la pregunta 4 del DAS ¿Cómo se siente cuando está en el sillón dental y ve que el dentista toma la máquina para empezar a trabajar en sus dientes?, un 30,2\% (51 personas) dijo estar tenso y solo un $18,9 \%$ (32 personas) manifestaron estar relajadas.

De un total de 169 adultos encuestados, el 60,8\% (103 personas) presentaron ansiedad dental. Según los diferentes niveles de $A D$ establecido por Corah ${ }^{(14)}$ se clasificaron los niveles de ansiedad en Tabla 2.

\section{Validez de Contenido}

Los expertos enviaron sus respuestas por correo electrónico, se analizaron sus juicios y comentarios; el $100 \%$ de los expertos consideró que la traducción, redacción y congruencia del instrumento de ansiedad dental (DAS) era adecuada para su aplicación a los acompañantes de niños que tienen atención clínica en los centros de atención primaria de la región Metropolitana. Finamente se reunieron los 4 expertos y se llegó al acuerdo que no era necesario realizar modificaciones al instrumento.

\section{Validez de Constructo}

Para chequear la pertinencia del análisis factorial confirmatorio, se calculó el estadístico de adecuación de la muestra de Kaiser-Meyer-Olkin (KMO) y la prueba de esfericidad de Bartlett. El coeficiente obtenido en la pueba KMO fue de 0,778 , mientras que en la prueba de esfericidad de Bartlett se obtuvo un valor de 238,63 con significancia menor al 0,05 (Tabla 3 ), siendo posible con estos resultados la realización del análisis factorial. Como se puede observar en la Tabla 4, este análisis confirmatorio arrojó un factor único con un autovalor de 2,618, que representa el $65,447 \%$ de la varianza

Para evidenciar la relación de los factores del DAS con la variable 
Validez de contenido, de constructo y confiabilidad del Dental Anxiety Scale en adultos chilenos.

Tabla 1. Variables sociodemográficas y fecha de última visita al dentista de adultos

\begin{tabular}{|c|c|c|}
\hline Variable & $\begin{array}{c}\text { Frecuencia } \\
(N=169)\end{array}$ & $\begin{array}{c}\text { Porcentaje } \\
(\%)\end{array}$ \\
\hline \multicolumn{3}{|l|}{ Edad } \\
\hline $18-39$ años & 111 & 65,7 \\
\hline 40-59 años & 48 & 28,4 \\
\hline Igual o mayor a 60 años & 10 & 5,9 \\
\hline \multicolumn{3}{|l|}{ Sexo } \\
\hline Femenino & 150 & 88,8 \\
\hline Masculino & 19 & 11,2 \\
\hline \multicolumn{3}{|l|}{ CESFAM } \\
\hline Recreo - San Miguel & 74 & 43,8 \\
\hline Bellavista - La Florida & 95 & 56,2 \\
\hline \multicolumn{3}{|l|}{ Nivel Educacional* } \\
\hline Básica incompleta & 6 & 3,7 \\
\hline Básica completa & 7 & 4,3 \\
\hline Media incompleta & 27 & 16,5 \\
\hline Media completa & 69 & 42,1 \\
\hline Superior incompleta & 21 & 12,8 \\
\hline Superior completa & 34 & 20,6 \\
\hline \multicolumn{3}{|c|}{ Fecha Última Visita AI Dentista } \\
\hline $6-12$ meses & 70 & 41,4 \\
\hline $1-2$ años & 37 & 21,9 \\
\hline $2-3$ años & 18 & 10,7 \\
\hline $3-4$ años & 5 & 3 \\
\hline $4-5$ años & 6 & 3,5 \\
\hline 5 años o más & 33 & 19,5 \\
\hline
\end{tabular}

Tabla 2. Clasificación Dental Anxiety Scale

\begin{tabular}{lccc}
\hline Resultado dental axiety scale & $\begin{array}{c}\text { Frecuencia } \\
(\mathbf{N}=169)\end{array}$ & $\begin{array}{c}\text { Porcentaje } \\
(\%)\end{array}$ \\
\hline 0 - 4 : No Ansioso & 66 & 39,2 \\
5 - 8 : Ansiedad Dental Leve & 53 & 31,3 \\
9 - 14 : Ansiedad Dental Moderada & 27 & 15,9 \\
15 - 20: Ansiedad Dental Severa & 23 & 13,6
\end{tabular}

Tabla 3. KMO y Prueba de Bartlett

\begin{tabular}{l|l|c}
\hline \multicolumn{2}{|l|}{$\begin{array}{l}\text { Medida de adecuación muestral de Kaiser - } \\
\text { Meyer - Olkin }\end{array}$} & 0,788 \\
\hline $\begin{array}{l}\text { Prueba de esfericidad } \\
\text { de Bartlett }\end{array}$ & $\begin{array}{l}\text { Chi - cuadrado } \\
\text { aproximado }\end{array}$ & 238,635 \\
\cline { 2 - 3 } & Significancia & $<0,05$ \\
\hline
\end{tabular}

Tabla 4. Varianza total explicada

\begin{tabular}{|c|c|c|c|}
\hline \multirow[t]{2}{*}{ Factor } & \multicolumn{3}{|c|}{ Autovalores iniciales } \\
\hline & Total & $\begin{array}{c}\% \text { de la } \\
\text { varianza }\end{array}$ & $\%$ acumulado \\
\hline 1 & 2,618 & 65,447 & 65,447 \\
\hline 2 & 0,540 & 13,505 & 78,952 \\
\hline 3 & 0,518 & 12,961 & 91,913 \\
\hline 4 & 0,323 & 8,087 & 100 \\
\hline
\end{tabular}

ansiedad dental, se obtuvo la matriz factorial (Tabla 5). El método de extracción utilizado fue máxima verosimilitud, se usó 1 factor y se requirieron 4 iteraciones. Los valores oscilan entre 0,654 correspondiente a la pregunta 4 y 0,827 correspondiente a la pregunta 1 .

Tabla 5. Matriz Factorial

\begin{tabular}{c|c}
\hline & Factor $\mathbf{1}$ \\
\hline 1 & 0,827 \\
\hline 2 & 0,796 \\
\hline 3 & 0,656 \\
\hline 4 & 0,654 \\
\hline
\end{tabular}

\section{Confiabilidad}

En el cálculo de la confiabilidad se obtuvo un coeficiente $\alpha$ de 0,822 considerado bueno ${ }^{(20,21)}$. Se aprecia una fuerte correlación entre cada uno de los ítems con el puntaje total, observando la correlación elemento-total corregida. Por último, al chequear la confiabilidad ítem-test, es posible apreciar que todos los ítems al ser eliminados provocan que el coeficiente de alfa de Cronbach disminuya, por lo que ninguno de ellos debiesen ser eliminados. (Tabla 6)

Tabla 6. Análisis confiabilidad

\begin{tabular}{c|c|c}
\hline Ítem & $\begin{array}{c}\text { Correlación elemento } \\
\text { - total corregida }\end{array}$ & $\begin{array}{c}\text { Alfa de cronbach si se } \\
\text { elimina elemento }\end{array}$ \\
\hline 1 & 0,601 & 0,797 \\
\hline 2 & 0,601 & 0,796 \\
\hline 3 & 0,681 & 0,760 \\
\hline 4 & 0,703 & 0,748 \\
\hline
\end{tabular}

La matriz de correlación, representada en la Tabla 7, indica la relación de cada ítem con los demás. Esta se realizó a través del coeficiente de correlación de Pearson $(r)^{(21)}$. La tabla muestra que todas las relaciones son positivas o directas, indicando que los ítems se encuentran relacionados entre sí.

Tabla 7. Matriz de correlaciones de Pearson

\begin{tabular}{c|c|c|c|c|c}
\hline \multicolumn{2}{c|}{ Ítem } & $\mathbf{1}$ & $\mathbf{2}$ & $\mathbf{3}$ & $\mathbf{4}$ \\
\hline \multirow{4}{*}{1} & $\begin{array}{c}\text { Correlación de } \\
\text { Pearson }\end{array}$ & 1 & $0,485^{*}$ & $0,492^{*}$ & $0,543^{*}$ \\
\cline { 2 - 6 } & Sig. (bilateral) & & $<0.05$ & $<0.05$ & $<0.05$ \\
\hline \multirow{2}{*}{2} & $\begin{array}{c}\text { Correlación de } \\
\text { Pearson }\end{array}$ & & 1 & $0,521^{*}$ & $0,515^{*}$ \\
\cline { 2 - 6 } & Sig. (bilateral) & & & $<0.05$ & $<0.05$ \\
\hline \multirow{3}{*}{3} & $\begin{array}{c}\text { Correlación de } \\
\text { Pearson }\end{array}$ & & & 1 & $0,671^{*}$ \\
\cline { 2 - 6 } & Sig. (bilateral) & & & & $<0.05$ \\
\hline \multirow{3}{*}{$\mathbf{4}$} & $\begin{array}{c}\text { Correlación de } \\
\text { Pearson }\end{array}$ & & & & 1 \\
\cline { 2 - 6 } & Sig. (bilateral) & & & & \\
\hline
\end{tabular}

Sig.: significancia

*. La correlación es significativa al nivel 0,05 (bilateral).

\section{DISCUSIÓN}

La versión en español del DAS ha sido usada en estudios realizados en Chile ${ }^{(9,10)}$, aunque ésta no ha sido validada en el país. En la presente investigación se evaluaron las propiedades psicométricas del intrumento de ansiedad dental DAS versión en idioma español.

En relación a la validez de contenido, a través de metodología Delphi, no hubo modificación alguna, puesto que cada experto consideró que el lenguaje utilizado, la redacción y la congruencia del DAS versión en 
idioma español, eran óptimas para su uso en el contexto cultural chileno.

En torno a la validez de constructo, el análisis factorial confirmatorio, arrojó un factor único con un autovalor de 2,618 que representó el $65,447 \%$ de la varianza total, lo que indica que este único factor es capaz de explicar gran parte del constructo ansiedad dental en la muestra evaluada. Los ítems del módulo DAS presentaron cargas o saturaciones de factor de 0,65 a 0,83 lo que indica una fuerte relación de cada pregunta con el constructo ansiedad dental. Estos resultados son similares a los obtenidos por Jain y Tandon ${ }^{(22)}$ con un autovalor de 2,441 que representó el $61,019 \%$ de varianza total.

Para el cálculo de la confiabilidad, a través de la consistencia interna, se obtuvo un coeficiente $\alpha=0,822$, el cual se considera como bueno(18,20,21). Este valor indica que el instrumento es homogéneo, es decir, todos los ítems del cuestionario miden ansiedad dental. El a obtenido en esta investigación fue similar a los encontrados en otros estudios de validación del DAS, como en Brasil(23) donde obtuvieron un $\alpha$ de 0,83 o en India(22), con un a de 0,782 .

Si se comparan los resultados obtenidos de la muestra estudiada, es posible indicar que la presencia de ansiedad dental en adultos chilenos que concurrieron a los servicios estudiados $(60,8 \%)$ es mayor a la de países como Alemania ${ }^{(6)}(40 \%)$, Noruega ${ }^{(5)}(16 \%)$, Australia( ${ }^{(7)}(31,2 \%)$ y Brasil $^{(8)}(24 \%)$, por lo tanto, la ansiedad dental es un factor importante a considerar al momento de realizar la atención dental integral, debido a que un gran porcentaje de personas presenta algún grado de ansiedad que influirá en el inicio y/o planificación del tratamiento dental $(6,12,13)$.

Un dato interesante es que frente a la pregunta de cuándo fue la última vez que visitó al odontólogo, un 19,5\% indicó que llevaba 5 años o más sin visitar al dentista. Estos datos se correlacionan con los reportados en la última Encuesta Nacional de Salud(24) realizada en Chile durante el 2017 , donde un $52,8 \%$ de los encuestados indicaron que hace más de 1 año que no visitaban al dentista. Si bien existen otros factores que influyen en el retraso y/o inasistencia a la consulta dental, los resultados obtenidos en este estudio podrían explicar en parte este hecho, ya que un alto nivel de ansiedad dental se asocia con un retraso a la visita al odontólogo (11).

El estudio presenta limitaciones al considerar las características de la muestra, al ser acompañantes de pacientes y al estar constituída mayormente por mujeres. La muestra es poco representativa pues sólo se estudiaron dos centros de atención primaria de la Región Metropolitana y los resultados podrían verse sesgados. Idealmente, próximos estudios debiesen considerar una muestra más equilibrada entre hombres y mujeres y aumentar el número de centros participantes a otras regiones del país. Por último, se debe considerar que los encuestados fueron adultos acompañantes de pacientes pediátricos. Resultaría interesante evaluar la influencia de este alto nivel de ansiedad dental del adulto en el comportamiento de los niños en el sillón dental, y ver la ansiedad dental de adultos que requieran atención odontológica.

Otra de las limitaciones del presente estudio es que solamente se utilizó la evaluación de la confiabilidad a través del método de consistencia interna alfa de Cronbach, por lo que sería recomendable para futuros estudios utilizar métodos adicionales para estimar la confiabilidad, como la técnica del test-retest.

A partir de los resultados obtenidos en esta investigación, se puede establecer que la versión en español del instrumento de ansiedad dental DAS representa una escala útil con sólidas propiedades psicométricas: valores adecuados de validez de contenido, validez de constructo y confiabilidad, para la recolección de información de ansiedad dental en la población de adultos que acuden a servicios de atención primaria en Chile.

\section{RELEVANCIA CLÍNICA}

Justificación científica para el estudio: La ansiedad dental influye en la salud oral, modificando pronóstico y adherencia a tratamiento. En Chile se utilizan cuestionarios no validados, por lo que resulta importante contar con un instrumento con buenas propiedades psicométricas.

Resultados Principales: El Instrumento Dental Anxiety Scale presentó una adecuada validez de contenido, validez de constructo y buena confiabilidad. Su uso es adecuado para evaluar ansiedad dental en adultos de la Región Metropolitana.

Consecuencias Prácticas: Tener esta escala con buenas propiedades psicométricas, permitiría tener una idea del nivel de ansiedad del paciente para direccionar el tratamiento de una manera más óptima.

\section{FUENTE DE FINANCIAMIENTO}

Este trabajo fue financiado por el Fondo Nacional en Salud (FONIS) dependiente de CONICYT, a través del Proyecto FONIS SA11I2025, titulado: "Impacto en la adherencia a tratamiento de una intervención cognitivo-conductual para disminuir ansiedad dental en niños y niñas de 6 años beneficiarios del programa de salud oral integral en servicios de atención primaria de la Región Metropolitana."

\section{CONFLICTO DE INTERÉS}

Los autores declaran no tener conflictos de interés.

\section{Bibliografía}

1. Ministerio de Salud (MINSAL), Subsecretaría de Redes Asistenciales División de Atención Primaria. Orientaciones para la implementación del modelo de atención integral de salud familiar y comunitaria. Dirigido a equipos de Salud. Santiago de Chile: Ministerio de Salud; 2013

2. Díaz Cárdenas S, Tirado Amador L, Vidal Madera A. Odontología con enfoque en salud familiar. Rev Cub Salud Pública. 2014;40(3):397-405.

3. Freeman R, Clarke H, Humphris G. Conversion tables for the Corah and Modified Dental Anxiety Scales. Community Dent Health. 2007;24(1):49-54.

4. Ríos Erazo M, Herrera Ronda A, Rojas Alcayaga G. Ansiedad dental: evaluación y tratamiento. Av Odontoestomatol. 2014;30(1):39-46.

5. Åstrøm A, Skaret E, Haugejorden O. Dental anxiety and dental attendance among 25-year-olds in Norway: time trends from 1997 to 2007. BMC Oral Health. 2011;11:10.

6. Eitner S, Wichmann M, Paulsen A, Holst S. Dental anxiety - an epidemiological study on its clinical correlation and effects on oral health. J Oral Rehabil. 2006;33(8):588-593.

7. Armfield J, Spencer A, Stewart J. Dental fear in Australia: who's afraid of the dentist?. Aust Dent J. 2006;51(1):78-85.

8. Carvalho R, Falcão P, Campos G, Bastos A, Pereira J, Pereira M et al. Ansiedade frente ao tratamento odontológico: prevalência e fatores predictores em brasileiros. Ciênc Saúde Coletiva. 2012;17(7):1915-1922.

9. Muza R, Muza P. Ansiedad en un servicio de emergencia dental. Horiz Enferm. 2008;19(1):23-28.

10. Ríos-Erazo $M$, Herrera-Ronda A, Barahona-Salazar $P$, Molina-Muñoz $Y$ Cadenasso-Salinas P, Zambrano-Canelo $\mathrm{V}$ et al. Ansiedad dental en adultos Chilenos que concurren a un servicio de salud primaria. Int $\mathrm{J}$ Odontostomat 2016;10(2):261-266

11. Pohjola V, Rekola A, Kunttu K, Virtanen J. Association between dental fear and oral health habits and treatment need among University students in Finland: a national study. BMC Oral Health. 2016;16:26
12. Bhola R, Malhotra R. Dental procedures, oral practices, and associated anxiety: A study on late-teenagers. Osong Public Health Res Perspect. 2014:5(4):219-232. 13. Seligman L, Hovey J, Chacon K, Ollendick T. Dental anxiety: An understudied problem in youth. Clin Psychol Rev. 2017;55:25-40.

14. Corah N. Development of a dental anxiety scale. J Dent Res. 1969;48(4):596596.

15. Newton J, Edwards J. Psychometric properties of the modified dental anxiety scale: an independent replication. Community Dent Health. 2005;22(1):40-42.

16. Caycedo C, Cortés O, Gama R, Rodríguez H, Colorado P, Caycedo M, et al. Ansiedad al tratamiento odontológico: características y diferencias de género. Suma Psicológica. 2008;15:259-278.

17. Armfield J. How do we measure dental fear and what are we measuring anyway? Oral Health Prev Dent. 2010;8(2):107-115.

18. Carvajal A, Centeno C, Watson R, Martínez M, Sanz Rubiales Á. ¿Cómo validar un instrumento de medida de la salud?. An Sist Sanit Navar. 2011; 34(1): 63-72.

19. Varela-Ruiz M, Díaz-Bravo L, Rocío G. Descripción y usos del método Delphi en investigaciones del área de la salud. Inv Ed Méd. 2012;1(2):90-95.

20. Martínez Ortega R, Leonel C T, Martínez Ortega M, Pérez Abreu A, Cánovas A. El coeficiente de correlación de los rangos de Spearman Caracterización. Rev Haban Cienc Méd. 2009;8(2).

21.Cronbach L. Coefficient alpha and the internal structure of tests. Psychometrika. 1951;16(3):297-334.

22. Jain M, Tandon S, Sharma A, Jain V, Rani Yadav N. Cross-Cultural adaption, validity and reliability of a Hindi version of the Corah's Dental Anxiety Scale. Health Promot Perspect. 2018;8(2):120-126.

23. Bonafé F, Campos J. Validation and invariance of the Dental Anxiety Scale in a Brazilian sample. Braz Oral Res. 2016;30(1):e138.

24. Ministerio de Salud (MINSAL). Encuesta Nacional de Salud ENS Chile 20092010. Santiago de Chile: Ministerio de Salud; 2015. 